\title{
O uso da terapia fotodinâmica na candidíase oral: Uma revisão de literatura
}

\author{
The use of photodynamic therapy in oral candidiasis: A literature review
}

Recebido: 29/10/2021 | Revisado: 03/11/2021 | Aceito: 08/11/2021 | Publicado: 11/11/2021

\author{
Giovana Dias Canteiro \\ Universidade Brasil, Brasil \\ E-mail: gigi_cante1@outllok.com \\ Camila Carnichelli Camargo \\ Universidade Brasil, Brasil \\ E-mail: camila.carnichelli@hotmail.com \\ Evellyn Gonçalo da Silva \\ Universidade Brasil, Brasil \\ E-mail: evellynsallo2@gmail.com \\ Juliana Rosa Soares Fernandes \\ Universidade Brasil, Brasil \\ E-mail: Juliana.rsf2000@gmail.com \\ Michele Cristina Silva do Vale \\ Universidade Brasil, Brasil \\ E-mail: neuromvale@gmail.com \\ André Chacon Montesino \\ Universidade Brasil, Brasil \\ E-mail: acmontesino@hotmail.com
}

\begin{abstract}
Resumo
A candidíase oral é a mais prevalente infecção fúngica encontrada na cavidade oral dos seres humanos. Mediante a possibilidade de resistência aos medicamentos antifúngicos, e seus efeitos colaterais, se torna necessário, o uso de novas práticas terapêuticas. A terapia fotodinâmica (PDT), se apresenta com bons resultados no controle, e tratamento da candidíase oral. O Objetivo deste estudo, é apresentar a importância da PDT, para o tratamento da candidíase oral. Método: revisão de literatura, caracterizada como descritiva e exploratória, a partir das bases de dados, MedLine, PubMed, Scielo, e Lilacs. Foram realizadas buscas em livros, artigos científicos, monografias, dissertações e teses, publicados entre 2011, e 2021. Os resultados sugerem que o uso da PDT, de maneira isolada possui potencial para o controle da candidíase oral de maneira satisfatória, todavia os melhores mais eficazes foram obtidos na associação da PDT, com a terapia convencional.
\end{abstract}

Palavras-chave: Candidíase Oral; Candida; Terapia fotodinâmica; Laser.

\begin{abstract}
Oral candidiasis is the most prevalent fungal infection found in the oral cavity of humans. Through the possibility of resistance to antifungal drugs, and their side effects. The use of new therapeutic practices becomes necessary. Photodynamic therapy (PDT) presents good results in the control and treatment of oral candidiasis. The aim of this study is to present the importance of PDT for the treatment of oral candidiasis. Method: literature review, characterized as descriptive and exploratory, based on MedLine, PubMed, Scielo, and Lilacs databases. Searches were carried out in books, scientific articles, monographs, dissertations and theses, published between 2011 and 2021. The results suggest that the use of PDT, in isolation, has the potential to satisfactorily control oral candidiasis, but the best ones more efficacious were obtained in the association of PDT with conventional therapy.
\end{abstract}

Keywords: Oral Candidiasis; Candida; Photodynamic Therapy; Laser.

\section{Introdução}

A candidíase oral é a mais prevalente infecção fúngica diagnosticada na cavidade oral dos seres humanos. Essa infecção fúngica abrange principalmente língua, palato, e a mucosa jugal dos indivíduos afetados. Têm etiologia diretamente relacionada a leveduras do gênero candida ssp. Dentre as espécies, a Candida albicans é considerada a mais deletéria. A candidíase é comumente descrita na literatura como uma infecção oportunista, onde a Candida ssp, abandona a relação de comensal, (onde duas espécies vivem juntas), e torna-se patogênica mediante as condições adequadas para sua proliferação, 
como por exemplo, xerostomia, diabetes mellitus, hipossalivação, uso prolongado de antibióticos, e corticóides, uso de próteses totais, e parciais. Por ser uma patologia oportunista, acredita-se que a Candidíase tenha uma relação direta com o sistema imunológico do paciente, ou seja, pacientes com o sistema imunológico debilitado, tem maiores chances de contrair esta infecção. Algumas doenças de ordem sistêmica, tem relação direta com a predisposição para o aparecimento da candidíase, como por exemplo, a síndrome da imunodeficiência adquirida, (AIDS), síndromes metabólicas, e neoplasias malignas. (Costa, 2011; Lescano et al, 2019; Nuñez et al, 2019; Teodoro et al, 2020; Rosa et al, 2021).

A ciência aponta três principais apresentações patológicas da candidíase oral: Candidíase eritematosa, candidíase pseudomembranosa, e hiperplásica. Na forma eritematosa, a lesão se apresenta na coloração vermelha, possui características dolorosas, com maior predisposição na região de dorso lingual contendo regiões despapiladas, e possui direta relação com o uso a longo prazo de antibióticos de longo espectro, que ao reduzirem o número de bactérias presentes na cavidade bucal, favorecem a proliferação da Candida ssp. A Candidíase pseudomembranosa, é caracterizada pelo surgimento de placas esbranquiçadas na região de mucosa bucal, prontamente removidas mediante a raspagem. Já a versão hiperplásica, é relatada pela alteração do tecido nas regiões de dorso da lingual, e comissura labial, tem coloração esbranquiçadas, não cedendo a raspagem. (Nünez et al, 2019; Teodoro, 2020; Rosa et al, 2021).

Os lasers têm se firmado cada vez mais como auxiliares no tratamento de patologias da cavidade bucal. A utilização do aparelho laser, para o tratamento de diversas doenças, possui registros catalogados desde a década de 60 . O laser, é um diodo, que emite luz, ou seja, um componente eletrônico que libera a passagem da eletricidade apenas em um único sentido, e necessita de pouca energia para a geração de luz. A literatura conceitua como terapia fotodinâmica, a combinação de uma fonte de luz, a um agente fotossensibilizador, quando o objetivo é a degradação e morte microbiana. A Terapia fotodinâmica abrange a união de um fotossensibilizador atóxico e uma matriz de luz evidente, na existência de oxigênio. (Eduardo et al, 2015; Queiroz, 2021).

Lasers de alta potência são comumente utilizados para a finalidade cirúrgica, promovendo remoção de tecido duro como, esmalte, dentina, e osso, além de vaporar o tecido mole, por meio de incisões. Possui ainda efeito antimicrobiano, que se dá pela elevação da temperatura durante o ato cirúgico. Já os lasers de baixa potência agem com efeito terapêutico, propiciando a reparação dos tecidos, modulação da sensação de dor, e diminuição de características de ordem inflamatória. Se tratando de lasers de baixa potência, não existe efeito antimicrobiano, visto que o aumento de tempetura tecidual não ocorre. Todavia, a taxa de redução microbiana associada a utilização de lasers de baixa potência está para 99-100\%, se relacionados a agentes fotossensibilizadores. (Eduardo et al, 2015; Queiroz, 2021).

Estudos apontam que a terapia fotodinâmica, é importante, e relevante para o tratamento, e controle da candidíase, visto que melhores resultados são obtidos quando existe a associação da PDT, com a terapia convencional. Essa prerrogativa é validada mediante a grande taxa de resistência aos fármacos, devido a pequena quantidade de antifúngicos disponíveis para o tratamento desta patologia, sustentado assim a ideia de necessidade de terapias complementares para o tratamento da candidíase. (Freitas, 2015, Lescano et al, 2019; Teodoro, 2020).

Dessa forma, este artigo tem por objetivo discorrer sobre a importância do uso da PDT como terapia para o tratamento da candidíase.

\section{Metodologia}

Este trabalho foi desenvolvido por meio de uma revisão de literatura baseada em livros, e artigos científicos indexados nas bases da Scientific Electronic Library Online (Scielo), US Nacional Library of Medicine National Institutes of Health (PubMed), Medical Literature Analysis and Retrieval System Online (Medline) e Literatura Latino-americana e do Caribe em Ciências da Saúde (Lilacs), com os descritores: Candidíase Oral; Cândida; Terapia fotodinâmica; e Laser, publicados nos 
últimos 10 anos. Os critérios de inclusão foram: artigos disponíveis na íntegra, e em português, publicados entre 2011, e 2021, relacionados com os descritores sugeridos. 32 artigos foram selecionados inicialmente para leitura do resumo e, 20 artigos foram lidos em sua totalidade para a composição do corpo desta revisão. (Estrela, 2018).

\section{Resultados e Discussão}

A PDT, é caracterizada como um tratamento que tem como base, o uso de luz, associada a fotossensibilizadores, a fim de promover morte celular através de um comprimento de onda específico, que possui a capacidade de ativar o agente fotossensível quando na presença de oxigênio. Essa proposta terapêutica, tem sido aplicada em várias especialidades da odontologia, permitindo procedimentos menos invasivos, assim como a estimulação de tecidos, e disseminação e microrganismos. (Cavalcanti, 2011; Ferreira et al, 2017; Reis, 2015).

O tratamento convencional da candidíase, é pautado na administração de medicamentos antifúngicos tópicos: nistatina, ou miconazol. A nistatina é o fármaco de primeira escolha, devido sua eficácia, e eficiência comprovadas para essa patologia, além de possuir menos efeitos colaterais, quando comparada a outros antifúngicos. Para pacientes portadores de diabetes mellitus, a nistatina, e o miconazol, são escolhas adequadas, pois em sua composição está presente a sacarina sódica, que tem poder de minimizar os riscos para esses pacientes. O fluconazol, cetoconazol, flucitosina, e anfotericina-B são os fármacos de escolha para o tratamento de forma sistêmica da candidíase. Em casos mais graves, é preconizado o uso de antofericina-B, ou equinocandinas de forma intravenosa. Contudo, ainda existe uma grande taxa de pacientes que adquirem resistência a essas medicações, promovendo um relevante aumento de recidivas. A terapia convencional, ainda possui como desvantagens, possíveis interações medicamentosas, náuseas, vômitos, e problemas gastrointestinais. (Siqueira et al, 2015, Teodoro et al, 2020).

A PDT, é um tratamento que tem por objetivo tratar infecções a nível superficial, e dessa forma não possui poder de destruição aos microrganismos nativos da flora bucal. Para que esse tratamento seja eficaz, é necessário o uso tópico de um fotossensibilizador, seguida de irradiação de luz com comprimento de onda específico, na presença de oxigênio. Após a ativação do fotossensibilizador mediante o uso da luz, algumas reações químicas a nível celular são produzidas, causando aumento da permeabilidade celular, causando danos as células fúngicas. Sendo considerada por Freitas, 2015, uma ótima estratégia de tratamento para a candidíase oral. (Nuñez et al, 2013; Teodoro et al, 2020).

Pereira et al. (2015), verificaram o potencial de inativação fotodinâmica, em 48 pacientes com candidíase, todos portadores de próteses removíveis, e com estomatite protética. Os resultados sugerem que após o tratamento com a PDT, somada a terapia convencional, houve uma redução considerável de algumas espécies de cândida, reduzindo assim a patogenicidade.

Alves 2013, avaliou os efeitos PDT, por meio do uso do Photodithazine ${ }^{\circledR}$ (PDZ) e luz LED, no tratamento da Candida albicans resistente ao antifúngico fluconazol em um modelo de candidose oral induzida em animais. A amostra foi composta por camundongos fêmeas com 6 semanas de vida, que inoculadas com C. Albicans, e imunossuprimidas. O Tratamento foi realizado apenas com LED, ou PDZ. Os resultados demonstram que a PDT, utilizada de forma isolada, apresenta boa resposta na redução de C. albicans, mesmo em animais resistentes ao fluconazol, não causando efeitos deletérios no tecido saudável.

Hidalgo (2018), verificou a relação do tratamento com PTD associada a nistatina em candidose oral induzida em camundongos inoculados com cândida, e resistentes a fluconazol. Diante dos achados macroscópicos, os resultados demonstram, uma boa diminuição das lesões na cavidade oral dos animais, seguida de redução da condição inflamatória, corroborando a ideia que a PDT, como terapia auxiliar, conjunta a terapia convencional, é eficaz em casos de candidose oral.

Afroozi e colaboradores (2019), apresentam um estudo onde investigam a eficácia da PDT, em combinação com a nistatina, para o tratamento e controle da estomatite dentaria, em comparação com a monoterapia utilizando nistatina somente. 
A amostra foi composta por 66 indivíduos, divididos em dois grupos. Grupo PDT associado a nistatina, e grupo nistatina. Ambos os grupos receberam tratamento 3 vezes por dia com nistatina, e o grupo PDT recebeu a terapia fotodinâmica uma vez na semana. Os resultados sugerem que a redução da colônia fúngica, e das lesões foi significativamente maior no grupo PDT associado a nistatina, quando comparado ao grupo que recebeu monoterapia com nistatina, não causando efeitos adversos. Reafirmando que a PDT, é uma interessante estratégia terapêutica para tratamento, quando comparado ao tratamento isolado com antifúngicos convencionais.

\section{Conclusão}

Diante dos estudos apresentados, acredita-se que a associação da PDT com a terapia convencional para o tratamento da candidíase oral, é mais eficaz, quando comparado a monoterapia convencional com antifúngicos tradicionais. Esses resultados demonstram que essa opção terapêutica possui eficiência, e deve ser considerada pelo profissional no plano de tratamento do paciente acometido por candidíase. A terapia conjunta entre PDT, e terapia convencional ainda demonstrou ser capaz de diminuir significativamente a quantidade de fungos, colaborando para uma remissão das lesões, e diminuição do processo inflamatório, o que colabora para o uso de uma menor quantidade de antifúngico, promovendo menor resistência a esse fármaco. Sugere-se mais estudos relacionando o uso da PDT de forma isolada em pacientes idosos hospitalizados com uso de tubotraqueal, visto que essa população, possui grande predisposição a ter candidíase oral.

\section{Referências}

Afroozi, B., Zomorodian, K, Lavaee, F, Shahrabadi, Z. Z, \& Mardani, M. (2019). Comparison of the efficacy of indocyanine green-mediated photodynamic therapy and nystatin therapy in treatment of denture stomatitis. Photodiagnosis and photodynamic therapy, 27, $193-197$.

Alves, F. (2013). Estudo in vivo dos efeitos da terapia fotodinâmica, mediada pelo Photothazine® e luz led, sobre Cândida Albicans resistente a Fluconazol.

Cavalcanti, T. M, Almeida-Barros, R. Q. D, Catão, M. H. C. D. V, Feitosa, A. P. A, \& Lins, R. D. A. U. (2011). Conhecimento das propriedades físicas e da interação do laser com os tecidos biológicos na odontologia. Anais Brasileiros de Dermatologia, 86, 955-960.

Costa, A. C. B. P. (2011). Avaliação da Terapia Fotodinâmica em Candida albicans in vitro e in vivo. 2011. Dissertação (Mestrado em Biopatologia Bucal)Faculdade de Odontologia de São José dos Campos, Universidade Estadual Paulista "Júlio de Mesquita Filho. https://repositorio.unesp.br/handle/11449/95047>.

Eduardo, C. D. P, Bello-Silva, M. S, Ramalho, K. M, Lee, E. M. R., \& Aranha, A. C. C. (2015). A terapia fotodinâmica como benefício complementar na clínica odontológica. Revista da Associação Paulista de Cirurgiões Dentistas, 69(3), 226-235.

Estrela, C. (2018). Metodologia científica: ciência, ensino, pesquisa. Artes Médicas.

Ferreira, C. L. R, Anacleto, F. N, Santos, C. H. D. S. D, Vieira, A. D, \& Vance, R. (2017). Uso de ultrassom e laser na descontaminação do sistema de canais radiculares: revisão de literatura. Dent. press endod, 27-33.

Freitas, L. S. D. F. (2015). Efeito antimicrobiano de múltiplas sessões de terapia fotodinâmica sobre biofilmes de Candida spp. formados in vitro.

Rimachi Hidalgo, K. J. (2018). Eficácia da terapia fotodinâmica antimicrobiana associada a nistatina no tratamento de candidose oral em camundongos infectados com Candida albicans resistente a fluconazol.

Lescano, F. A, Oliveira P. T, Vieira, I. P, Oliveira, J. H. M, da Costa, M. W, Juliano, F. M. S, \& Simões, E. A. P. (2019). Utilização da terapia fotodinâmica em candidíase oral. Perspectivas Experimentais e Clínicas, Inovações Biomédicas e Educação em Saúde (PECIBES), 5(2), 67-67.

Nunez, S., Garcez, A. S., \& Ribeiro, M. S. (2015). PDT-Terapia fotodinâmica antimicrobiana na odontologia. Elsevier Brasil.

Nuñez, S. C, Ribeiro, M. S, Garcez, A. S. (2019). PDT - Terapia fotodinâmica antimicrobiana na odontologia. (2a ed.), Elsevier. 253.

Oliveira, R. F, Silva, L. P. D. L, Silva, F. V. D, Andrade, K. D. S, Moreira Romão, T. C, Dos Santos, M. G. C, \& Arnaud, R. R. (2021). Terapia fotodinâmica associada a laser no tratamento endodôntico. Archives of health investigation, 10(2), 236-240. https://doi.org/10.21270/archi.v10i2.5051.

Pereira, C. A, Domingues, N, Silva, M. P, Costa, A. C. B. P, Junqueira, J. C, \& Jorge, A. O. C. (2015). Photodynamic inactivation of virulence factors of Candida strains isolated from patients with denture stomatitis. Journal of Photochemistry and Photobiology B: Biology, 153, 82-89.

Queiroz, R. S. (2021). Aplicação da terapia fotodinâmica no tratamento endodôntico: Revisão de literatura.

Reis, F. (2015). Tecnologias endodônticas. Santos. 
Rosa, C, Curi, V, Rosa, A, Filho, A, Bianchi, C, Deps, T, Crepaldi, M, Crepaldi, M, \& Crepaldi, M. (2021). Candidíase bucal. Revista Faipe, 11(1), 155-163.

Siqueira, J. S, Batista, S. A, Silva Jr, A., Ferreira, M. F, Agostini, M, \& Torres, S. R. (2015). Candidíase oral em pacientes internados em UTI. Revista Brasileira de Odontologia, 71(2), 176.

Sousa, B. J. L., Nascimento, J. A., Motta, M. N. A., Rocha, G. C. E. S., Moura, A. L., Jesus, G. P., Silva, K. M. (2021). Terapia fotodinâmica para o tratamento das lesõe orais: revisão de literatura. Brazilian Journal of Development.7(6), 56229-56246.

Teodoro, P, \& Fernandes, H. V. (2020). O uso da terapia fotodinâmica como método alternativo de tratamento da candidíase oral. Revista Arquivos Científicos (IMMES), 3(1), 14-23. https://doi.org/https://doi.org/10.5935/2595-4407/rac.immes.v3n1p14-23. 